\title{
Synthesis of Superporous Hydrogels by a Postpolymerization Foaming Protocol and Their Water Absorbent Behavior
}

\author{
Ruiying Gong, Ning Kang, Youbing Mu, Jianzhong Li, Xiaobo Wan \\ Qingdao Institute of Bioenergy and Bioprocess Technology, Chinese Academy of Sciences, Qingdao 266101, China
}

Received 26 July 2010; accepted 1 November 2011

DOI 10.1002/app.36441

Published online 1 February 2012 in Wiley Online Library (wileyonlinelibrary.com).

\begin{abstract}
The rate of swelling is one of the most important parameters of hydrogel polymeric networks; therefore, the objective of this study was to develop a new method to synthesize superporous poly(acrylic acid-coacrylamide) hydrogels to improve swelling rate. The hydrogel was prepared in the presence of a foaming agent (comprising azodicarbonamide and zinc oxide), using 2,2'Azobis[2-(2-imidazolin-2-yl)propane] dihydrochloride as the initiator. The $\mathrm{N}_{2}$ released during the polymerization led to the formation of a slightly foamed gel; we refer to the process as a prefoaming process. The obtained gel underwent a further foaming process to form a superporous hydrogel. Unlike in existing methods, the consistent time control of the foaming agent addition and the onset
\end{abstract}

of gelling were not necessary in this simplified gel preparation procedure. The water absorbent behavior of the superporous hydrogels suggested that the polymers obtained from the prefoaming process reached the swelling equilibrium more rapidly than those with little prefoaming, and their absorbent capacity was greater. Scanning electron microscope images show that interconnected pores inside these superporous gels formed capillary channels, which are critical for the fast swelling rate. () 2012 Wiley Periodicals, Inc. J Appl Polym Sci 125: 31003106, 2012

Key words: superporous hydrogels; prefoaming process; swelling; absorbent capacity

\section{INTRODUCTION}

Super absorbent hydrogels are lightly cross-linked networks of hydrophilic polymers that absorb water and swell into many times their original size and weight. They are widely used in many fields, such as sanitary products, agricultural, and horticultural water-holding materials. Fast swelling rate of the hydrogels is preferred in applications such as gastric retention devices and sanitary products. Acrylicacrylamide (AM)-based superabsorbent hydrogels are mostly used, but it often takes a few hours for these hydrogels to fulfill the swelling equilibrium. ${ }^{1-6}$ To increase the rate of absorption, capillary channels formed by interconnected pores are needed in the superabsorbent hydrogels. Superporous hydrogels are different from superabsorbent polymers in their fast swelling rate, their ability to reach the equilibrium within minutes regardless of their size. As far as we know, porous hydrogels can be prepared by many different methods, such as porosigen technique, ${ }^{7-9}$ phase separation technique, ${ }^{10-13}$ crosslinking of individual polymer particles, ${ }^{14}$ and gas blowing (or foam-

Correspondence to: R. Gong (gongry@qibebt.ac.cn).

Journal of Applied Polymer Science, Vol. 125, 3100-3106 (2012) (C) 2012 Wiley Periodicals, Inc. ing) technique. ${ }^{15}$ The major disadvantages of those methods have been described in detail by other research groups. ${ }^{16}$ Recently, it was reported that porous hydrogels could be synthesized by crosslinking polymerization of various vinyl monomers in the presence of gas bubbles formed by the chemical reaction of an acid and $\mathrm{NaHCO}_{3}{ }^{16,17}$ The obtained porous hydrogels could swell in aqueous solution in a few minutes regardless of their size and shape, due to the presence of capillary channels inside the gels.

However, a lot of these methods include two processes, that is, gelation and foaming processes. Those two processes need to be conducted in such a way to enable harmonized foaming and gelation, ${ }^{18}$ which means the timing for foaming agent addition and the onset of gelling must be controlled carefully, so that the gas bubbles could be captured by the polymer matrix and distributed evenly inside the matrix. Otherwise, if the foaming process began too early, the bubbles would be released from the solution before the onset of gelling, and no capillary channel would be formed; if the foaming process began too late, the solution would become too viscous for the even distribution of gas bubbles. The former led to a poorly porous or sometimes nonporous hydrogel, and the latter led to a nonhomogenous one.

Being aware of these drawbacks, we developed a new method to synthesize superporous hydrogels. 


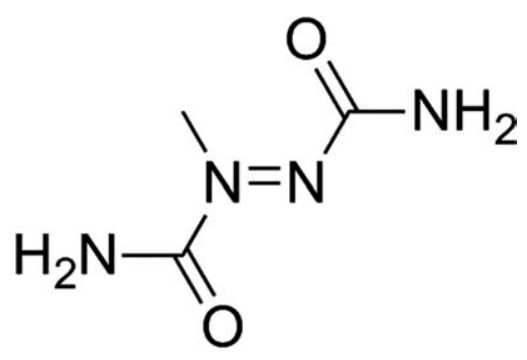

Figure 1 The structure of AC.

Inspired by the molding technique of foam materials such as rubber foams and plastic foams, we used an azodicarbonamide (AC) foaming agent, which is comprised of $\mathrm{AC}$ and zinc oxide $(\mathrm{ZnO})$, as the bubble resources in the hydrogel synthesis. AC (structure shown in Fig. 1) decomposes to release $\mathrm{N}_{2}$ at elevated temperature, and $\mathrm{ZnO}$ acts as the catalyst to decrease the decomposition temperature of $\mathrm{AC}$. AC foaming agent is the mostly used and effective foaming agent for plastics and rubbers because of its low toxicity and adjustable decomposition temperature. The foaming agent was predispersed in the monomer solution before polymerization. After the polymerization, the foaming agent-containing polymer was further treated by a foaming process to form a superporous hydrogel. Because the foaming agent was preadded and only decomposed to form bubbles during the postpolymerization foaming process, the consistent time control of the foaming agent addition and the onset of gelling was avoided, which simplified the gel preparation process.

\section{EXPERIMENTAL}

\section{Materials}

Acrylic acid (AA), AM, N,N'-methylenebisacrylamide (MBS), sodium hydroxide, and potassium persulfate (KPS) were purchased from Sinopharm Chemical Reagent Company. 2,2'-Azobis[2-(2-imidazolin-2-yl)propane] dihydrochloride (AIBI) was purchased from China Qindao Runxing Photoelectric Material Company. AC foaming agent was purchased from China Qindao Sainuo Chemical Company.

\section{Synthesis of superporous hydrogels}

Superporous hydrogels were synthesized according to a reported procedure. ${ }^{19} \mathrm{~A}$ typical synthesis of superporous hydrogels was shown below: $24 \mathrm{~mL}$ of AA solution (40 wt \%, with $\mathrm{pH}$ value adjusted to 56), $40 \mathrm{~mL}$ of distilled water, $0.06 \mathrm{~g}$ of MBS, $0.6 \mathrm{~g}$ of $\mathrm{AC}$, and $8.0 \mathrm{~g}$ of $\mathrm{AM}$ were added in sequence into a round-bottom flask with vigorous magnetic stirring to form a well-dispersed mixture. The mixture was purged with $\mathrm{N}_{2}$ for $20 \mathrm{~min}$ at $65^{\circ} \mathrm{C}$, followed by the addition of $0.01 \mathrm{~g}$ of AIBI. Gel formation was generally observed in $10 \mathrm{~min}$. The reaction was maintained at this temperature for $4 \mathrm{~h}$. The obtained polymer was fully foamed under warm air blowing at $165^{\circ} \mathrm{C} \pm 2{ }^{\circ} \mathrm{C}$ for $1 \mathrm{~h}$, and then dried for a day with warm air blowing at $85^{\circ} \mathrm{C} \pm 2{ }^{\circ} \mathrm{C}$. A dried resin was obtained without further treatment. The product was smashed to get a powdered superporous resin.

\section{Absorbency tests under unload conditions}

To measure the absorbent capacity, $1.0 \mathrm{~g}$ sample of the resin powder (20-100 mesh) was immersed in $200 \mathrm{~mL}$ of tap water or in $0.9 \mathrm{wt} \% \mathrm{NaCl}$ solution for $\sim 3 \mathrm{~h}$ to reach the swelling equilibrium. Each of the swollen samples under unload conditions was filtered through a 100-mesh sieve, and the excess water on the surface was removed carefully from the water-containing gel. The absorbent capacity of the resin $(Q)$ in tap water or in 0.9 wt $\% \mathrm{NaCl}$ solution was determined as follows:

$$
Q=\left(M-M_{0}\right) / M
$$

where $M$ is the mass of the final gel and $M_{0}$ is the mass of the dried resin.

\section{Analysis}

Thermo gravimetric analysis (TGA) and differential scanning calorimetry (DSC) were carried out on Thermogravimetry-1100SF (METELLO TOLEDO Co.) to study the decomposition temperatures of the dried resin powder. Small samples (about 2-3 mg) were encapsulated in aluminum pans and analyzed in a temperature range from $30^{\circ} \mathrm{C}$ to $800^{\circ} \mathrm{C}$ at a heating rate of $10^{\circ} \mathrm{C} / \mathrm{min}$ in nitrogen atmosphere.

The macrostructure of hydrogel was studied with steromicroscopy-SF2 (Japan). The water-containing gel samples were directly divided into $2 \mathrm{~cm} \times 4 \mathrm{~cm}$ $\times 2 \mathrm{~cm}$ sizes without drying.

The morphology of the porous structure of the resin was studied with scanning electron microscopy (SEM). The dried resin powder was coated with a thin layer of gold and imaged in a Cambridge S-4800 SEM instrument.

\section{RESULT AND DISCUSSION}

\section{Effects of AIBI}

In this study, we first chose KPS as the initiator. Generally, in the reported procedure, when the concentration of KPS was $0.3 \mathrm{wt} \%$ and the total concentration of monomers $\mathrm{AA}$ and $\mathrm{AM}$ was $28.5 \mathrm{wt} \%$, the polymerization occurred smoothly. However, in our case, no gel was formed even after the reaction 


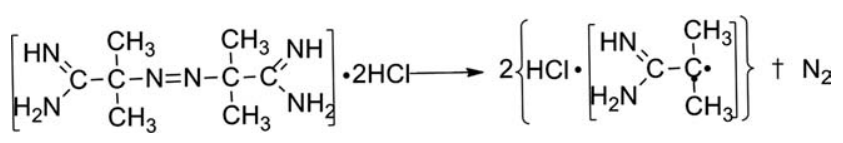

Figure 2 The AIBI initiation mechanism.

was carried out at $65^{\circ} \mathrm{C}$ for a day at the same KPS concentration, which indicated that the polymerization did not occur. This might be due to the presence of $\mathrm{AC}$ foaming agent in the reaction mixture, which lowed the initiating efficiency of the KPS. When we switched to water-soluble AIBI as the initiator, much better polymerization was observed. We are not sure the real reason for this, but given the similarity between the structure of AIBI and AC, it is reasonable to postulate that AC would not interfere with the initiation of the AIBI. AIBI decomposition mechanism was shown in Figure 2.

The concentration of AIBI played an important role on the gelation rate. Figure 3 shows the correlation between the gel formation time and AIBI concentration. The gelation time was shortened with the increase of AIBI concentration. When the concentration of AIBI was above $0.67 \mathrm{wt} \%$, the gel formation was observed in around $10 \mathrm{~min}$.

More interestingly, AIBI acted not only as an initiator, but also as a foaming agent. The polymerization and foaming process occurred almost simultaneously, due to the $\mathrm{N}_{2}$ release during the decomposition of AIBI in the initiation process. A slightly foamed gelatin polymer was obtained, even without the later foaming process. We called this a prefoaming process.

An obvious macrostructure change of the gelatin polymer with a prefoaming process was observed in compared with the one without a prefoaming process, as shown in Figure 4. When AIBI concentration was 0.20 wt $\%$, there resulting polymer showed a macrostructure with sparsely distributed bubbles [Fig. 4(1a)], mainly due to the presence of air dissolved in the reaction solution. When AIBI concentration was increased to $0.67 \mathrm{wt} \%$, more $\mathrm{N}_{2}$ was released during the polymerization, and much more abundant gas bubbles were observed in the polymer, as shown in Figure 4(1b). This facilitated the formation of even more abundant gas bubbles during later foaming process, as observed in Figure $4(2 b)$, in comparison with that in Figure 4(2a).

The prefoaming process also led to a faster swelling rate of the final resulting polymers. Figure 5 showed the influence of AIBI concentration on the swelling time and the absorbent capacity of the resin. Regardless of AIBI concentration, all the superporous hydrogels swelled rapidly in tap water, and reached swelling equilibrium within $10 \mathrm{~min}$. When the concentration of AIBI was above 0.67 wt \%, it took around 5 min to fulfill the swelling equilibrium. When the con- centration of AIBI was below 0.67 wt \%, it took around $10 \mathrm{~min}$ to reach the swelling equilibrium. A similar trend was observed in 0.9 wt \% $\mathrm{NaCl}$ solution as shown in Figure 5(b). Furthermore, we examined the swelling rate of the poly(AA-co-AM) hydrogels without undergoing a foaming process. It took $52 \mathrm{~min}$ in tap water and $74 \mathrm{~min}$ in 0.9 wt $\% \mathrm{NaCl}$ solution to reach the equilibrium under the same test conditions. This indicated that the porous hydrogels obtained after the foaming process swell much faster than common superabsorbent polymers. This swelling rate was in the same range with that of superporous hydrogels. ${ }^{20-22}$

The influence of the AIBI concentration on the absorbent capacity of the final polymer was, however, more complicated. In general, the absorbent capacity of a hydrogel polymer is in proportion with the molecular weight, hence in inverse proportion with the concentration of the initiator. However, a rebounce of the absorbent capacity was observed with the increase of the initiator concentration in our case. The largest absorbent capacity (Q) was 192.9 and in tap water and 59.51 in 0.9 wt \% $\mathrm{NaCl}$ solution, respectively, as shown in Figure 5. This largest capacity did not appear at the lowest concentration of AIBI (0.03 wt \%) but at $0.4 \mathrm{wt} \%$. At higher concentrations, although there was a decrease of the polymer absorbent capacity, it was still better than that of the polymer obtained at low concentrations. Given that the polymer obtained at higher AIBI concentrations had more porous structure due to a better prefoaming process, this unusual result inferred that the prefoaming process could compensate the loss of absorbent capacity caused by the increase of AIBI concentration, and sometimes, even improve the absorbent capacity to some extent.

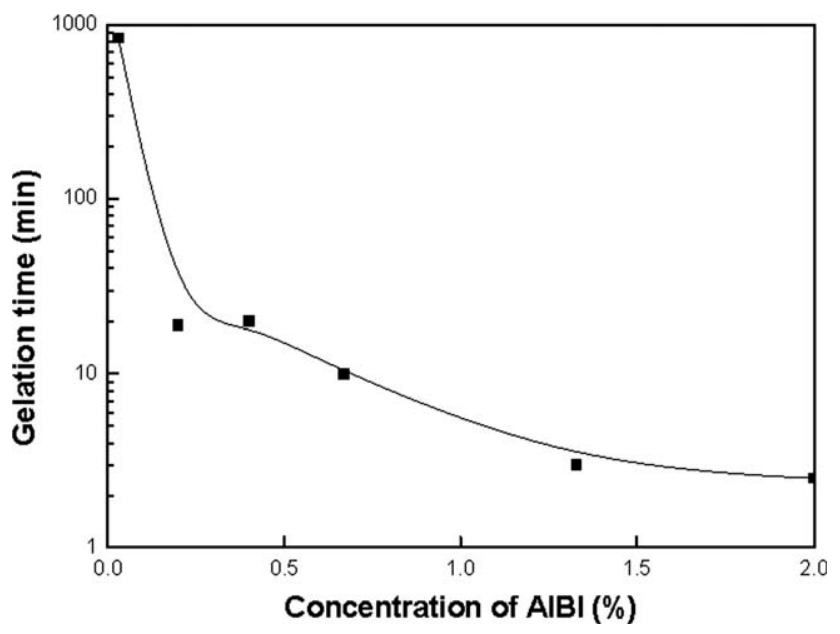

Figure 3 The influence of AIBI concentration on the gelation time. The amounts of MBS and AC were fixed at 0.06 $\mathrm{g}$ and $0.3 \mathrm{~g}$, respectively, in all cases. 

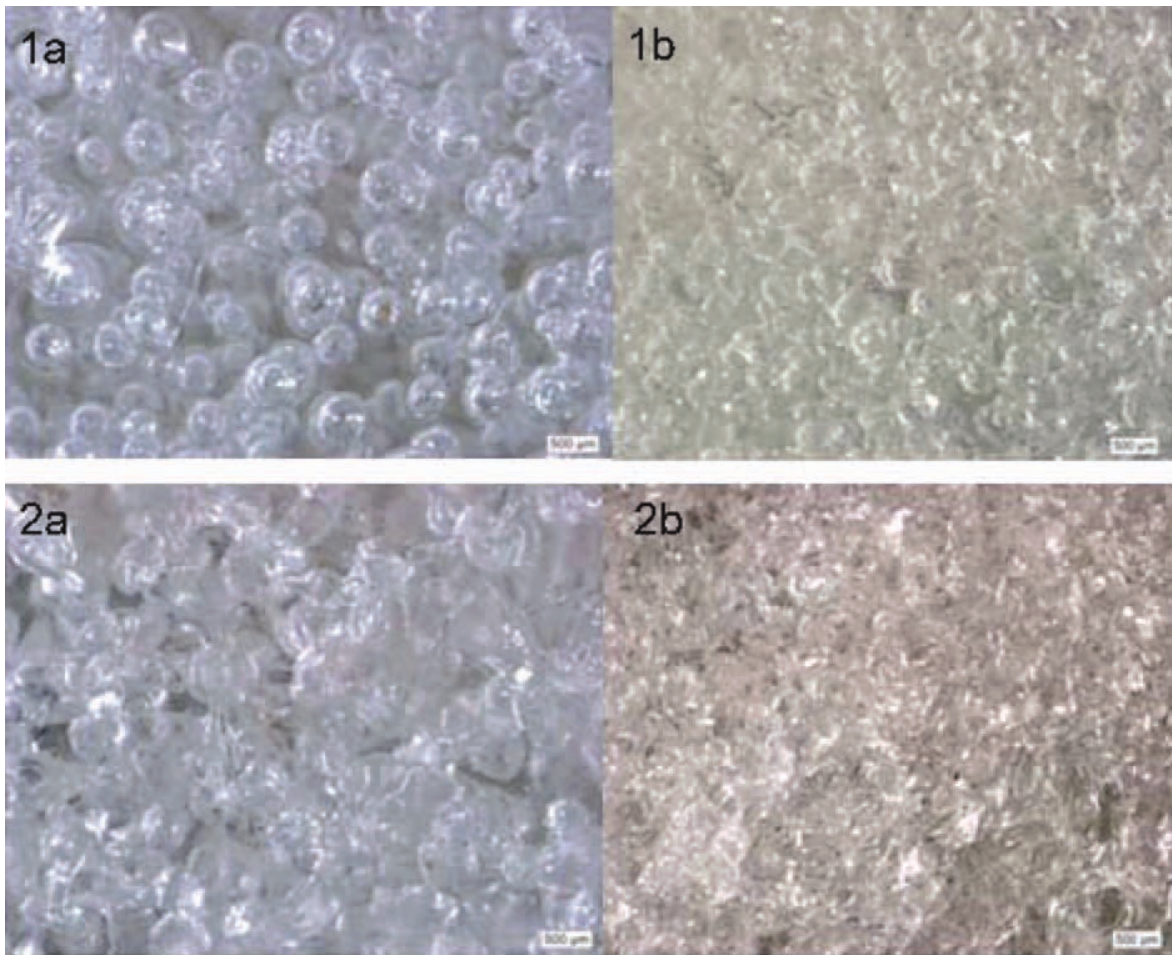

Figure 4 The photographs of the macrostructure of gelatin polymers prepared in different conditions: (1a) gelation polymers formed with little prefoaming process and $(1 \mathrm{~b})$ with a full prefoaming process, both before postpolymerization foaming process; ( $2 \mathrm{a}$ and $2 \mathrm{~b}$ ) gelatin polymers obtained after postpolymerization foaming process from samples (1a and 1b), respectively. [Color figure can be viewed in the online issue, which is available at wileyonlinelibrary.com.]

\section{Influence of $\mathrm{AC}$ foaming agent}

In the postpolymerization foaming process, the foaming temperature would influence the quality of the resulting hydrogel. Pure AC starts to decompose around $205^{\circ} \mathrm{C}$, and $\mathrm{ZnO}$ are added to decrease its decomposition temperature in commercially available AC foaming agents. ${ }^{23,24}$ We screened three AC

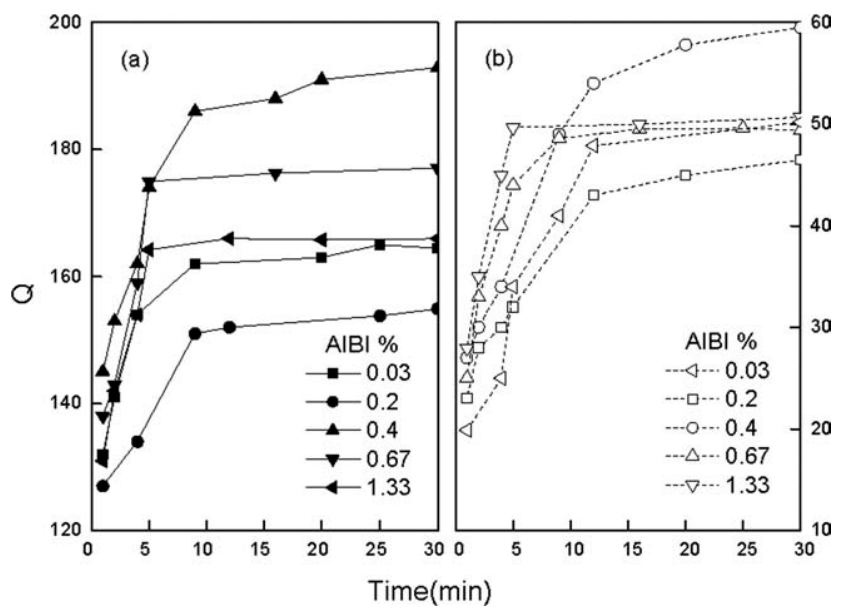

Figure 5 The absorbent capacity $(Q)$ and swelling rate of superporous hydrogels as a function of AIBI concentration: (a) in tap water; (b) in 0.9 wt $\% \mathrm{NaCl}$ solution. The amounts of MBS and AC were fixed at $0.06 \mathrm{~g}$ and $0.3 \mathrm{~g}$, respectively, in all cases. foaming agents with decomposing temperatures at $120^{\circ} \mathrm{C}, 160^{\circ} \mathrm{C}$, and $200^{\circ} \mathrm{C}$, respectively. Our preliminary studies showed that when the decomposing temperature of $\mathrm{AC}$ was $120^{\circ} \mathrm{C}$, a small proportion of AC would decompose and release $\mathrm{N}_{2}$ during the polymerization, which decreased the foaming efficiency in the postpolymerization foaming process. This was due to the fact that the polymerization was exothermic, which might lead to a local temperature jump to over $120^{\circ} \mathrm{C}$, and cause the partial decomposition of AC. So the decomposition temperature should not be lower than $120^{\circ} \mathrm{C}$. On the other hand, when the decomposing temperature of $\mathrm{AC}$ was $200^{\circ} \mathrm{C}$, however, a color change of superporous hydrogels took place during the postpolymerization foaming process, which decreased the quality of the hydrogels. TGA and DSC results in Figure 6 verified that the decomposition of the polymer matrix started to occur when temperature rose beyond $200^{\circ} \mathrm{C}$. From $50^{\circ} \mathrm{C}$ to $200^{\circ} \mathrm{C}$, only a mass loss due to the vaporization of volatile components was observed regardless of the content of AC. In the corresponding DSC curves, no evidential peaks were observed in this temperature region, suggesting no structural damage in the material. When the temperature exceeded $200^{\circ} \mathrm{C}$, a more rapid mass loss was observed, suggesting that the polymeric material started to decompose, which was accompanied by a color change of superporous hydrogels. In the consideration of these 


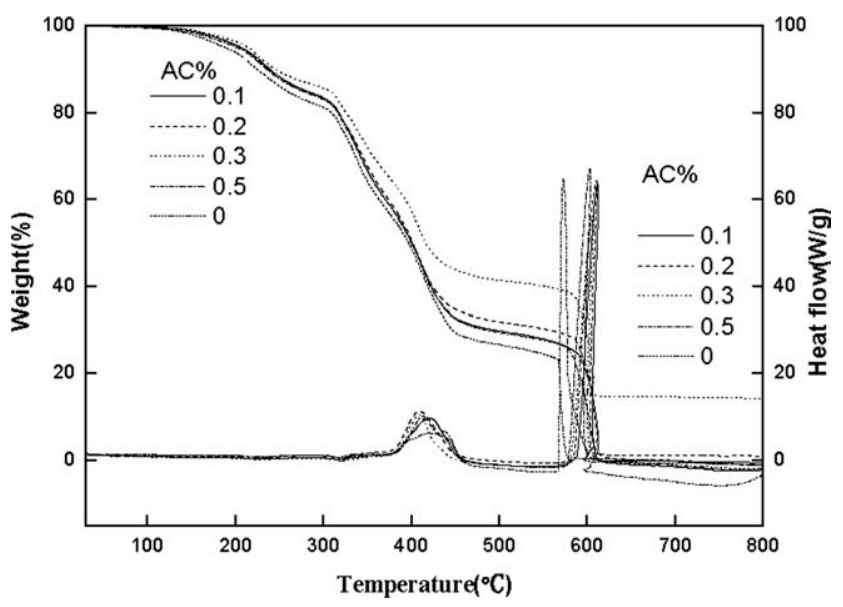

Figure 6 TGA and DSC analysis of the dried resin powder as a function of $\mathrm{AC}$ concentration. The amounts of MBS and AIBI were fixed at $0.06 \mathrm{~g}$ and $0.03 \mathrm{~g}$, respectively, in all cases.

two factors, we chose the foaming agent AC with a decomposing temperature of $160^{\circ} \mathrm{C}$ as the best agent for foaming.

The concentration of AC had a great influence on gelation rate. Gelation rate was a key factor for the prefoaming process. When gelation rate became slow, the formation of polymer matrix would be too slow to capture the bubbles generated from AIBI decomposition. The curves in Figure 7 revealed that the gelation onset was postponed with the increase of the concentration of AC. When the concentration of $\mathrm{AC}$ was below $0.3 \mathrm{wt} \%$, the gelation started to occur in less than $10 \mathrm{~min}$, which was favorable for the prefoaming process. When the concentration of AC was above 0.42 wt $\%$, the gelation was not observed until the reaction time reached $100 \mathrm{~min}$.

Figure 8 showed the influence of AC concentration on the swelling rate. When the concentration of $\mathrm{AC}$

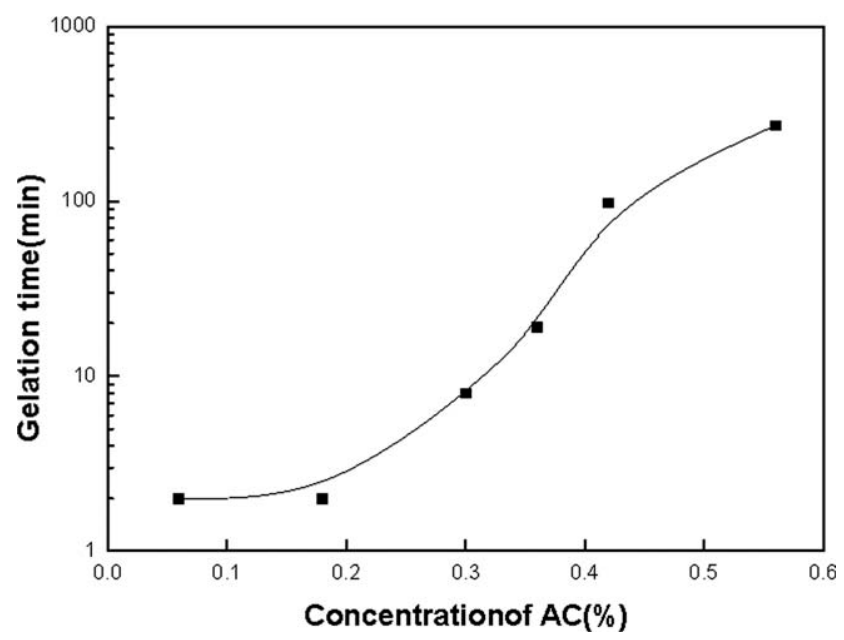

Figure 7 The influence of AC concentration on the gelation time. The amounts of MBS and AIBI were fixed at $0.06 \mathrm{~g}$ and $0.03 \mathrm{~g}$, respectively, in all cases.

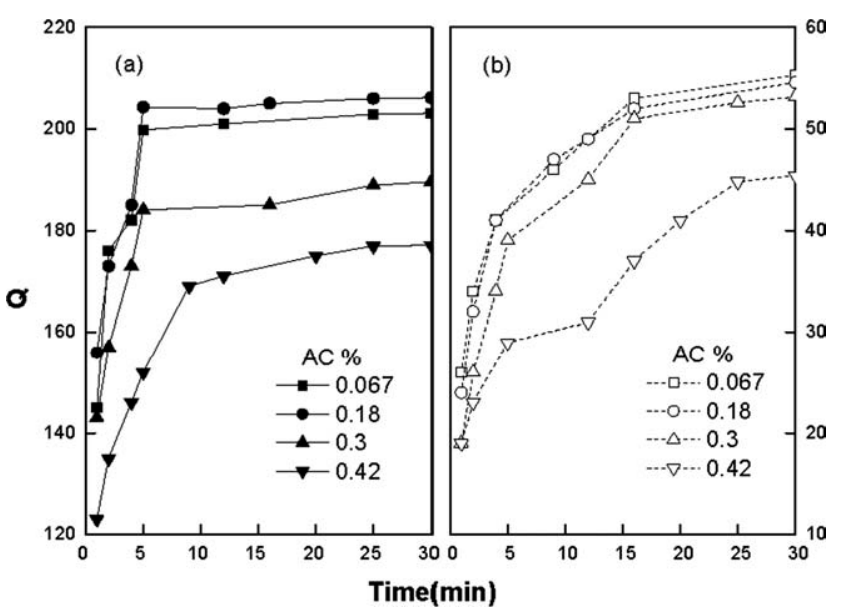

Figure 8 The absorbent capacity $(Q)$ and swelling rate of superporous hydrogels as a function of AC concentration: (a) in tap water; (b) in $0.9 \mathrm{wt} \% \mathrm{NaCl}$ solution. The amounts of MBS and AIBI were fixed at $0.06 \mathrm{~g}$ and $0.03 \mathrm{~g}$, respectively, in all cases.

was below $0.3 \mathrm{wt} \%$, it took around $5 \mathrm{~min}$ to fulfill the swelling equilibrium in tap water. Although the concentration of $\mathrm{AC}$ was above $0.42 \mathrm{wt} \%$, it took more than $10 \mathrm{~min}$ to reach the swelling equilibrium. Similar swelling rate change with regard to AC concentration was observed in 0.9 wt $\% \mathrm{NaCl}$ solution. This suggested a better prefoaming process led to a better foamed hydrogel, which has a faster swelling rate compared with that of the poly(AA-co-AM) hydrogels obtained without a foaming process.

Figure 8 also revealed the correlation of the absorbent capacity of the hydrogel and the AC concentration. In lower AC concentration region (lower than $0.18 \%$ ), the absorbent capacity of the hydrogel either increased slightly [in tap water, Fig. 8(a)], or remained almost unaffected [in $0.9 \% \mathrm{NaCl}$ solution, Fig. 8(b)] with the increase of the AC concentration. At higher AC concentrations (beyond $0.18 \%$ ), the absorbent capacity dropped, both in tap water and in $0.9 \% \mathrm{NaCl}$ solution. It showed that the increase of AC concentration did not favor the polymerization, so did not favor the improvement of the absorbent capacity.

Given all the factors discussed above, the optimized AC concentration for the best absorbent capacity was $0.18 \mathrm{wt} \%$.

\section{Influence of MBS}

The crosslinker (MBS) concentration also had a great influence on the gelation rate. As shown in Figure 9, higher concentration of MBS led to shorter gelation time. When the concentration of MBS was below $0.0067 \mathrm{wt} \%$, the onset of gelation was more than $100 \mathrm{~min}$. When the concentration of MBS was above $0.03 \mathrm{wt} \%$, the gelation started within $5 \mathrm{~min}$. 


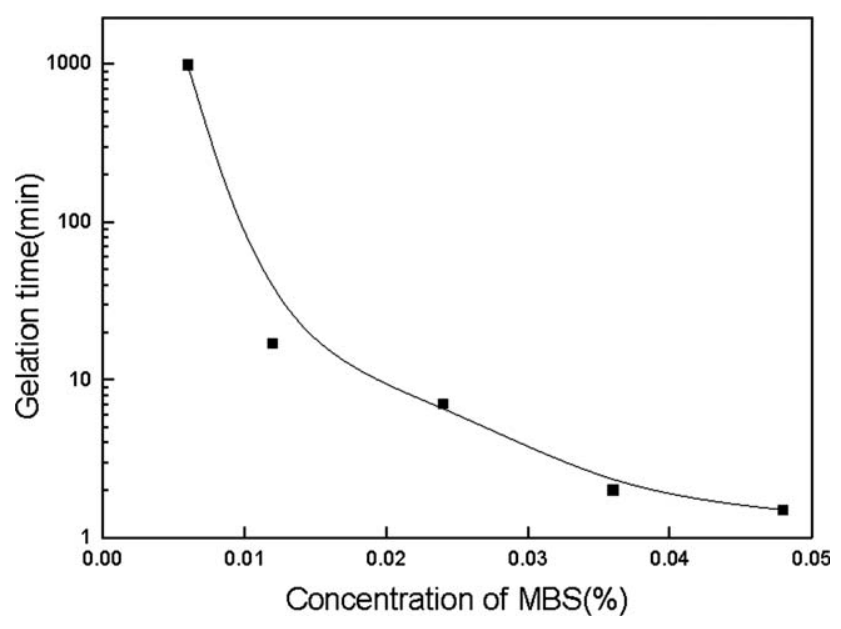

Figure 9 The influence of MBS concentration on the gelation time. The amounts of AIBI and AC were fixed at $0.03 \mathrm{~g}$ and $0.3 \mathrm{~g}$, respectively, in all cases.

From the data of swelling rate in Figure 10, we could draw the same conclusion: the porous hydrogen obtained with a better prefoaming process had an improved swelling rate compared with that of the poly(AA-co-AM) hydrogels without undergoing a foaming process.

The influence of MBS concentration on the absorbent capacity was also investigated. Normally, crosslinking density increases with the increase of the crosslinker concentration, which lowers the absorbent capacity of the hydrogel. ${ }^{25,26}$ However, we found a reversed result in our case. As shown in Figure 10, the hydrogel with the largest absorbent capacity was obtained when the MBS concentration was at $0.03 \%$. Lower crosslinker concentration led to a worse performance. This again was correlated to the faster gelation rate at higher crosslinker concentrations, which favored a better prefoaming process.

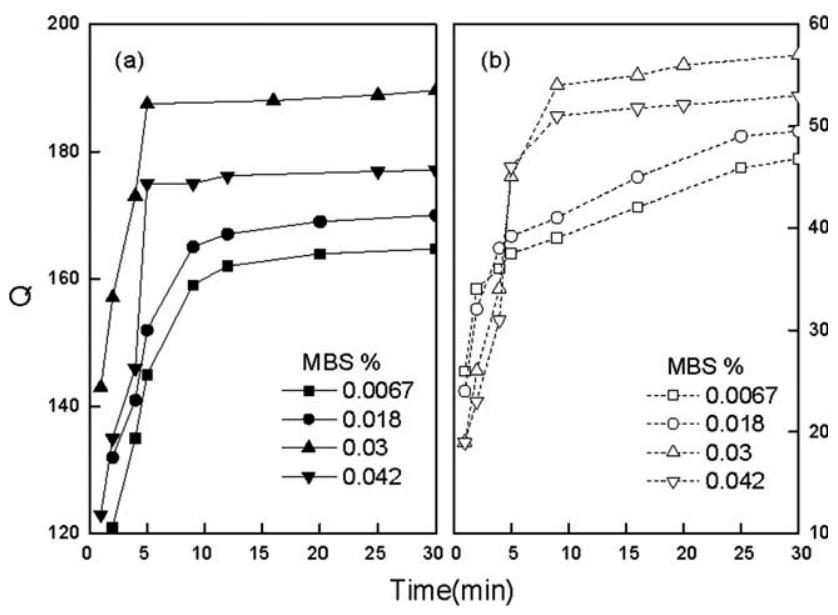

Figure 10 The absorbent capacity $(Q)$ and swelling rate of superporous hydrogels as a function of MBS concentration: (a) in tap water; (b) in $0.9 \mathrm{wt} \% \mathrm{NaCl}$ solution. The amounts of AIBI and AC were fixed at $0.03 \mathrm{~g}$ and $0.3 \mathrm{~g}$, respectively, in all cases.
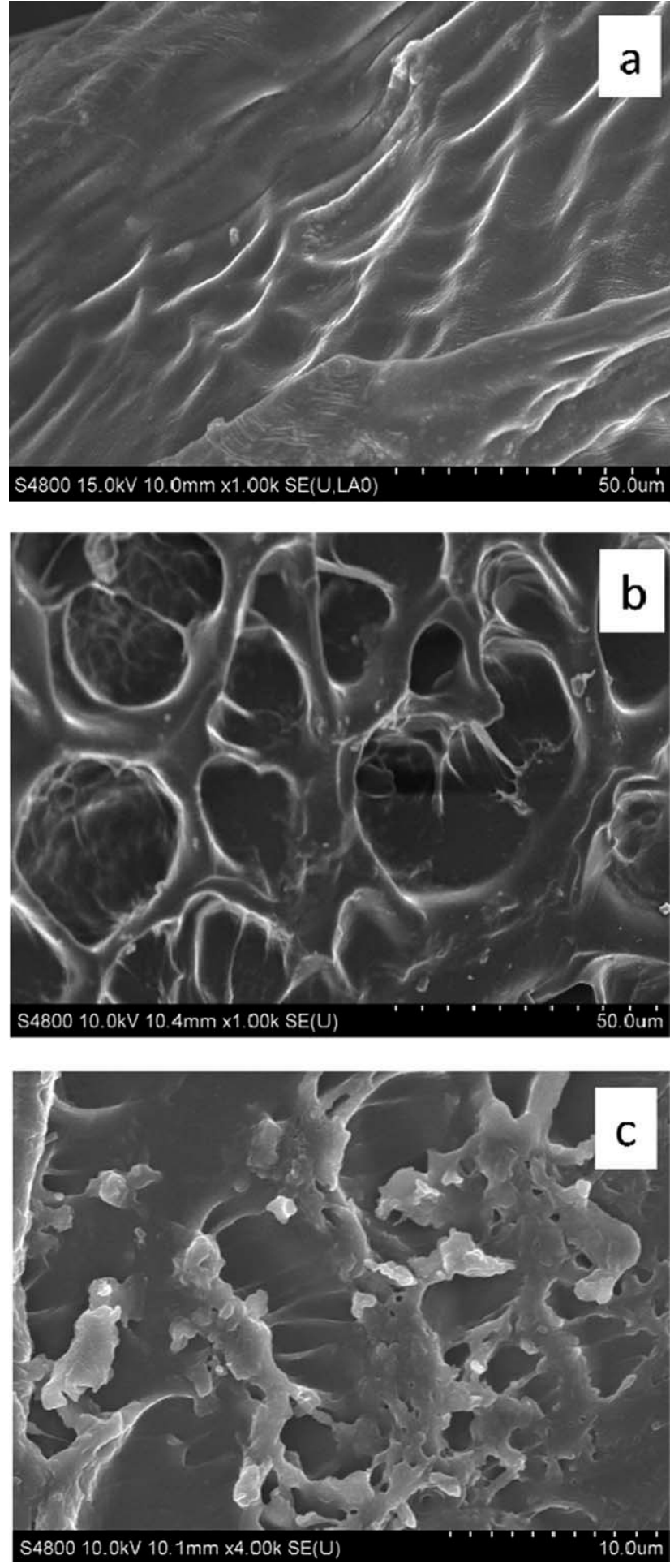

Figure 11 SEM photographs of superporous hydrogels at different magnification level.

\section{SEM images of porous structure}

The microscopic images of the dried nonporous hydrogels and superporous hydrogel gave a visual evidence of their inner structure, as shown in Figure 11. Interconnected pores could not be observed for an air-dried $\mathrm{P}(\mathrm{AM}-\mathrm{co}-\mathrm{AA})$ without foaming process 
[Fig. 11(a)]. For an air-dried P(AM-co-AA) superabsorbent hydrogel with the foaming process [Fig. $11(b, c)]$, interconnected pores with a average diameter in 10-50 $\mu \mathrm{m}$ range was observed, which was beyond the pores size defined by "microporous' (10-100 nm range) and "macroporous' (100 nm to $10 \mu \mathrm{m}$ range) superabsorbent polymers. ${ }^{7,10}$ We called the gel prepared this way a "superporous' hydrogel. ${ }^{16}$ SEM images also showed that capillary channels formed by interconnected pores, which we believe were critical for fast swelling rate.

\section{CONCLUSIONS}

We report herein a new method to synthesize superporous and superabsorbent hydrogels. This method does not need to control the consistency of timing for addition of the foaming agent and the onset of gelling carefully, which simplified the process of preparing superporous hydrogel. The porous hydrogel obtained could swell within minutes to the equilibrium swollen compared with a few hours of no porous hydrogels and was in accordance with the reported data about superporous hydrogels.

These results indicate that the polymers obtained from a combination of the prefoaming process during polymerization and the following-up foaming process reach swelling equilibrium more rapidly than those of without such a process and also improve the swelling rate and the absorbent capacity to some extent. The concentrations of the initiator, foaming agent, and crosslinker had a great influence on the performing process and the properties of the hydrogel, which was discussed in detail. By the discussion about the absorbent ability and swelling rate of porous hydrogels obtained, it can be decided the porous hydrogels obtained as a kind of superporous and superabsorbent hydrogels, which will be good for application in sanitary products and agriculture.

\section{References}

1. Mohan, Y. M.; Murthy, P. S. K.; Raju, K. M. J Appl Polym Sci 2006, 101, 3202.

2. Wan, T.; Yao, J.; Ma, X. J Appl Polym Sci 2008, 110, 3859.

3. Zhang, Y.; Wang, L.; Li, X.; He, P. J Polym Res 2010, 18, 157.

4. Ma, Z.; Li, Q.; Yue, Q.; Gao, B.; Xu, X.; Zhong, Q. Bioresour Technol 2011, 102, 2853.

5. Zhou, W.; Zhang, Y.; Jin, K.; Qiu, X.; Ren, X.; Hu, S.; Zhang, F. J Appl Polym Sci 2009, 114, 2828.

6. Seetapan, N.; Srisithipantakul, N.; Kiatkamjornwong, S. Polym Eng Sci 2011, 51, 764.

7. Oxley, H. R.; Corkhill, P. H.; Fitton, J. H.; Tighe, B. J. Biomaterials 1993, 14, 1064.

8. Badiger, M. V.; McNeill, M. E.; Graham, N. B. Biomaterials 1993, 14, 1059.

9. Krauch, C. H.; Sanner, A. Naturwissenschaften 1968, 55, 539.

10. Chirila, T. V.; Constable, I. J.; Crawford, G. J.; Vijayasekaran, S. Biomaterials 1993, 14, 26.

11. Kabra, B. G.; Gehrke, S. H. Polym Commun 1991, 32, 322.

12. Kabra, B. G.; Gehrke, S. H. In Superabsorbent PolymersScience and Technology; Buchholz, F. L., Peppas, N. A., Eds.; Washington, DC: American Chemical Society, 1994.

13. Yan, Q.; Hoffman, A. S. Polymer 1995, 36, 887.

14. Buchholz, F. L. In Superabsorbent Polymers-Science and Technology; Buchholz, F. L., Peppas, N. A., Eds.; Washington, DC: American Chemical Society, 1994.

15. Zamani, A.; Henriksson, D.; Taherzadeh, M. J. Carbohydr Polym 2010, 80, 1091.

16. Chen, J.; Park, H.; Park, K. J Biomed Mater Res 1999, 44, 53.

17. Chen, J.; Park, K. J Controlled Release 2000, 65, 73.

18. Omidian, H.; Rocca, J. G.; Park, K. J Controlled Release 2005, $102,3$.

19. Wang, W. B.; Wang, A. Q. Carbohydr Polym 2010, 80, 1028.

20. Salimi, H.; Pourjavadi, A.; Seidi, F.; Jahromi, P. E.; Soleyman, R. J Appl Polym Sci 2010, 117, 3228.

21. Pourjavadi, A.; Soleyman, R.; Barajee, G. R. Starch-Stärke 2008, 60, 467.

22. Chen, J.; Park, H.; Park, K. J Biomed Mater Res 1999, 44, 53.

23. Marcilla, A.; Garcia-Quesada, J. C.; Beltran, M. I.; Ruiz-Femenia, R. J Appl Polym Sci 2008, 107, 2028.

24. Zhang, B. S.; Lv, X. F.; Zhang, Z. X.; Liu, Y.; Kim, J. K.; Xin, Z. X. Mater Des 2010, 31, 3106.

25. Tang, Q. W.; Sun, X. M.; Li, Q. H.; Lin, J. M.; Wu, J. H. J Mater Sci 2009, 44, 3712.

26. Yarimkaya, S.; Basan, H. J Macromol Sci Pure Appl Chem 2007, 44, 939. 